\title{
An important step in establishing a treatment strategy for small renal masses of clear cell renal cell carcinoma based on the significance of adverse histopathologic features on tumor needle biopsy
}

\author{
Suguru Kadomoto", Tomoyuki Makino ${ }^{\#}$, Kouji Izumi, Atsushi Mizokami \\ Department of Integrative Cancer Therapy and Urology, Kanazawa University Graduate School of Medical Science, Kanazawa, Japan \\ "These authors contributed equally to this work. \\ Correspondence to: Kouji Izumi, MD, PhD. Department of Integrative Cancer Therapy and Urology, Kanazawa University Graduate School of \\ Medical Science, 13-1 Takara-machi, Kanazawa, Ishikawa 920-8641, Japan. Email: azuizu2003@yahoo.co.jp. \\ Provenance: This is an invited article commissioned by the Section Editor Dr. Xiao Li (Department of Urology, Jiangsu Cancer Hospital \& Jiangsu \\ Institute of Cancer Research \& Affiliated Cancer Hospital of Nanjing Medical University, Nanjing, China). \\ Comment on: Yang C, Shuch B, Serrano M, et al. Adverse Histopathologic Characteristics in Small Clear Cell Renal Cell Carcinomas Have Negative \\ Impact on Prognosis: A Study of 631 Cases With Clinical Follow-up. Am J Surg Pathol 2019;43:1413-20.
}

Submitted Oct 17, 2019. Accepted for publication Dec 16, 2019.

doi: 10.21037/atm.2019.12.104

View this article at: http://dx.doi.org/10.21037/atm.2019.12.104

Renal cell carcinoma (RCC) accounts for $3-4 \%$ of all adult cancer cases in the United States, and is divided into two groups: clear cell RCC (CCRCC) and non-CCRCC $(1,2)$. CCRCC is the most dominant histological phenotype that accounts for $70-80 \%$ of all RCC cases. A total of $20-40 \%$ of patients with RCC experience metastatic or recurrent disease after a primary surgery [nephrectomy or partial nephrectomy $(\mathrm{PN})$ ], and approximately $50 \%$ of patients with RCC eventually receive systemic medication during their disease course (2). Patients with CCRCCs presenting with small renal masses (SRMs), defined as contrast-enhancing kidney tumor with a maximum diameter of $\leq 4 \mathrm{~cm}$, generally show long-term survival with indolent and dormant features (3). Recently, the number of incidentally diagnosed SRMs without any symptoms and metastases has increased because of the widespread use of imaging examinations and technological development of diagnostic imaging (4). Therefore, various treatment options have been available for patients with CCRCC and SRMs, including active surveillance (AS); ablative therapies such as cryoablation, radiofrequency ablation, and high-intensity focused ultrasound; PN with minimum incision, endoscopic, laparoscopic, and robot- assisted laparoscopic surgery; and radical nephrectomy (RN) (5). Optimal management is required in such cases with the consideration of oncologic outcomes, such as tumor volume control, disease-free survival (DFS), diseasespecific survival (DSS), and overall survival (OS), and potential adverse events associated with each treatment option. A study evaluating RCC patients aged $\geq 75$ years showed that SRM interventions were unassociated with OS improvement (6). Increasing age and comorbidity were associated with death from any cause with cardiovascular disorders as the most common (6). Patel et al. reported that differences in OS and DSS were statistically insignificant among RN, PN, and AS for SRMs with a median followup of 34 months (7). A recent multi-institutional study enrolling 497 patients with SRMs who selected either primary active intervention or AS reported that OS for surgical intervention and AS was $98 \%$ and $96 \%$ at 2 years and $92 \%$ and $75 \%$ at 5 years, respectively $(\mathrm{P}=0.06)$, and DSS for primary intervention and AS at 5 years was $99 \%$ and $100 \%$, respectively $(\mathrm{P}=0.3)$ (8). AS was not predictive of OS or DSS in regression modeling with a relatively short follow-up. Based on these results, AS could be considered as a safe management option for select patients with SRMs. 
Hence, AS has been widely recommended for renal tumors with diameter $\leq 3 \mathrm{~cm}$, increasing diameter $<5 \mathrm{~mm}$ yearly, no infiltration, low complexity, and favorable histopathology (including chromophobe type RCC and oncocytoma) per the American Urological Association guideline. AS is being recognized as a standard of care for the management of SRMs (9). However, histology-based studies that stratify the prognosis of patients with SRMs have been limited, in addition to the lack of prospective and long-term outcomes; furthermore, existing studies have been largely limited to the grade and histologic subtype. An accurate detection system is required for aggressive CCRCCs because some SRMs show a highly aggressive disposition resulting in local invasion and distant metastasis during AS.

Therefore, Yang et al. conducted a study for determination of the extent and frequency of adverse histopathologic features in CCRCCs of $\leq 4 \mathrm{~cm}$ in size and their relevance to patient outcomes (10). They reviewed the pathological archives of RCC patients in their hospital for identification of radical and partial nephrectomies carried out and found 631 consecutive cases of nephrectomies (10). Adverse histopathologic characteristics were identified because they are known to correlate with the prognosis of patients with RCC. They were necrosis, high nuclear grade defined as International Society of Urological Pathology (ISUP)/World Health Organization (WHO) grades 3 and 4, lymphovascular invasion, sarcomatoid, and rhabdoid histology defined as $>5 \%$ of sarcomatoid or rhabdoid morphological tumor cells. However, information on SRM is still lacking (11). Their study indicated that tumor size is strongly correlated with both the presence and percentage of high nuclear grade (10). Additionally, patients with at least one adverse histologic finding patients had a $84.7 \%$ 5 -year DFS and $82.9 \% 10$-year DFS, in contrast to the 5 -year DFS of $95.3 \%$ and 10 -year DFS of $95.3 \%$ in patients without adverse histology $(\mathrm{P}=0.0043)(10)$. The 5 - and 10-year OS for patients with at least one adverse histologic finding were $90.2 \%$ and $90.2 \%$, respectively, whereas for those without adverse histology were $97.8 \%$ and $91.1 \%$, respectively (10). Although the difference in OS was insignificant between patients with and without any adverse histology, a marginally significant difference was observed ( $\mathrm{P}=0.0554)$ (10). These data indicate that a specific subpopulation of patients with CCRCC and SRM requires invasive treatment options, such as ablative therapies, $\mathrm{PN}$, and $\mathrm{RN}$ in some cases.

As mentioned by the authors in the paper, adverse histopathologic characteristics should be accurately diagnosed before deciding the course of treatment. Tumor needle biopsy seems to be the only method for histopathologic diagnosis at pretreatment. Nondiagnostic biopsies have been reported to be $0-21 \%$, and the coincidence between diagnosis based on biopsy and nephrectomy pathology was $86.7-100 \%(12-15)$. A recent systematic review summarizing the current outcomes of tumor needle biopsy reported a median overall diagnostic rate of $92 \%$, and sensitivity and specificity of tumor needle biopsy against surgical pathology were $99.1 \%$ and 93.2\%, respectively (16). Moreover, in another systematic review, core biopsy showed high sensitivity [97.5\%, 95\% confidence interval (CI), 96.5-98.5] and specificity (96.2\%; 95\% CI, 90.7-100) when a pathological diagnosis was obtained; however, the overall nondiagnostic rate was $14.1 \%$. Importantly, Fuhrman upgrading in surgical pathology was notable (16\%) from low [1-2] to high grade [3-4] (17). The tumor grade differences between biopsy and surgical pathology results may be considered when performing tumor needle biopsy. Although the most controversial complication of tumor needle biopsy may be the potential risk of tumor seeding along the needle tract, the overall estimated risk was reported to be $<0.01 \%$ (18). A most recent study reported that tumor needle biopsy was strongly and independently associated with reduced surgery for SRMs, especially with increasing age and comorbidity (19). Based on these results, tumor needle biopsy may be a reasonable diagnostic method before treatment for SRMs. However, the tumor needle biopsy procedure should be appropriately performed to obtain adequate amount of tissue. Wunderlich et al. recommended that one central and one peripheral biopsy specimen should be obtained from tumors $<4 \mathrm{~cm}$ and two peripheral specimens from larger tumors (20). It is especially challenging to detect the presence of necrosis, lymphovascular invasion, and rhabdoid or sarcomatoid component because these pathologic findings are usually observed in small limited areas.

Objective scoring methods without tumor needle biopsy to differentiate patients with SRMs are most suitable for $\mathrm{AS}$, such as the delayed intervention and surveillance for small renal mass (DISSRM) score (age, ECOG performance status, highest tumor diameter, RENAL nephrometry score, dementia, or cardiovascular index), has been reportedly useful for guiding the management selection (21). As an additional workup such as confirmatory biopsy should be 
performed to achieve an accurate treatment decision, tumor needle biopsy may be replaced with these scoring methods in the future.

The present study is expected to be an important step in establishing a treatment strategy for SRMs of CCRCC based on the significance of adverse histopathologic features on tumor needle biopsy. Further investigations with a large sized, prospective, and randomized design must confirm the importance of adverse histopathologic characteristics in patients with SRMs and their association with long-term outcomes.

\section{Acknowledgments}

None.

\section{Footnote}

Conflicts of Interest: The authors have no conflicts of interest to declare.

Ethical Statement: The authors are accountable for all aspects of the work in ensuring that questions related to the accuracy or integrity of any part of the work are appropriately investigated and resolved.

\section{References}

1. Siegel RL, Miller KD, Jemal A. Cancer statistics, 2019. CA Cancer J Clin 2019;69:7-34.

2. Molina AM, Motzer RJ. Current algorithms and prognostic factors in the treatment of metastatic renal cell carcinoma. Clin Genitourin Cancer 2008;6 Suppl 1:S7-13.

3. Gill IS, Aron M, Gervais DA, et al. Clinical practice. Small renal mass. N Engl J Med 2010;362:624-34.

4. Nguyen MM, Gill IS, Ellison LM. The evolving presentation of renal carcinoma in the United States: trends from the Surveillance, Epidemiology, and End Results program. J Urol 2006;176:2397-400; discussion 400.

5. Ljungberg B, Bensalah K, Canfield S, et al. EAU guidelines on renal cell carcinoma: 2014 update. Eur Urol 2015;67:913-24.

6. Lane BR, Abouassaly R, Gao T, et al. Active treatment of localized renal tumors may not impact overall survival in patients aged 75 years or older. Cancer 2010;116:3119-26.

7. Patel N, Cranston D, Akhtar MZ, et al. Active surveillance of small renal masses offers short-term oncological efficacy equivalent to radical and partial nephrectomy. BJU Int 2012;110:1270-5.

8. Pierorazio PM, Johnson MH, Ball MW, et al. Five-year analysis of a multi-institutional prospective clinical trial of delayed intervention and surveillance for small renal masses: the DISSRM registry. Eur Urol 2015;68:408-15.

9. Campbell S, Uzzo RG, Allaf ME, et al. Renal Mass and Localized Renal Cancer: AUA Guideline. J Urol 2017;198:520-9.

10. Yang C, Shuch B, Serrano M, et al. Adverse Histopathologic Characteristics in Small Clear Cell Renal Cell Carcinomas Have Negative Impact on Prognosis: A Study of 631 Cases With Clinical Follow-up. Am J Surg Pathol 2019;43:1413-20.

11. Delahunt B, Cheville JC, Martignoni G, et al. The International Society of Urological Pathology (ISUP) grading system for renal cell carcinoma and other prognostic parameters. Am J Surg Pathol 2013;37:1490-504.

12. Hara I, Miyake H, Hara S, et al. Role of percutaneous image-guided biopsy in the evaluation of renal masses. Urol Int 2001;67:199-202.

13. Lechevallier E, Andre M, Barriol D, et al. Fine-needle percutaneous biopsy of renal masses with helical CT guidance. Radiology 2000;216:506-10.

14. Caoili EM, Bude RO, Higgins EJ, et al. Evaluation of sonographically guided percutaneous core biopsy of renal masses. AJR Am J Roentgenol 2002;179:373-8.

15. Shah RB, Bakshi N, Hafez KS, et al. Image-guided biopsy in the evaluation of renal mass lesions in contemporary urological practice: indications, adequacy, clinical impact, and limitations of the pathological diagnosis. Hum Pathol 2005;36:1309-15.

16. Marconi L, Dabestani S, Lam TB, et al. Systematic Review and Meta-analysis of Diagnostic Accuracy of Percutaneous Renal Tumour Biopsy. Eur Urol 2016;69:660-73.

17. Patel HD, Johnson MH, Pierorazio PM, et al. Diagnostic Accuracy and Risks of Biopsy in the Diagnosis of a Renal Mass Suspicious for Localized Renal Cell Carcinoma: Systematic Review of the Literature. J Urol 2016;195:1340-7.

18. Volpe A, Kachura JR, Geddie WR, et al. Techniques, safety and accuracy of sampling of renal tumors by fine needle aspiration and core biopsy. J Urol 2007;178:379-86.

19. Patel HD, Nichols PE, Su ZT, et al. Renal Mass Biopsy is Associated with Reduction in Surgery for Early-Stage Kidney Cancer. Urology 2019. [Epub ahead of print].

20. Wunderlich H, Hindermann W, Al Mustafa AM, et al. 
The accuracy of 250 fine needle biopsies of renal tumors. J Urol 2005;174:44-6.

21. Sotimehin AE, Patel HD, Alam R, et al. Selecting Patients with Small Renal Masses for Active Surveillance: A Domain Based Score from a Prospective Cohort Study. J Urol 2019;201:886-92.

Cite this article as: Kadomoto S, Makino T, Izumi K, Mizokami A. An important step in establishing a treatment strategy for small renal masses of clear cell renal cell carcinoma based on the significance of adverse histopathologic features on tumor needle biopsy. Ann Transl Med 2019;7(Suppl 8):S374. doi: $10.21037 /$ atm.2019.12.104 